\title{
Influencia de la secuenciación de contenidos y el apoyo parental en la motivación de estudiantes de violín en edad preescolar ${ }^{1}$
}

\author{
Influence of Sequencing of Contents and the Parental Support \\ on the Motivation of Preschool Violin Students
}

\author{
Roberto Macián-González (1) https://orcid.org/0000-0002-5765-617X \\ Jesús Tejada Giménez (2) https://orcid.org/0000-0003-0532-3960 \\ (1) Universidad Jaume I \\ (2) Universidad de Valencia \\ (Recibido: 13 de febrero de 2018; Aceptado para su publicación: 21 de mayo de 2018)
}

Cómo citar: Macián-González, R. y Tejada, J. (2020). Influencia de la secuenciación de contenidos y el apoyo parental en la motivación de estudiantes de violín en edad preescolar. Revista Electrónica de Investigación Educativa, 22, e07, 1-14. https://doi.org/10.24320/redie.2020.22.e07.2257

\section{Resumen}

La secuenciación de contenidos y el papel de la familia pueden ser cuestiones fundamentales en iniciación instrumental, influyendo de manera decisiva en la motivación de los alumnos. Esta investigación analizó esta influencia en la iniciación al violín con 11 estudiantes en edad preescolar en clase individual. Para ello, se llevó a cabo una investigación-acción realizada por el profesor como observador participante, utilizando dos aproximaciones didácticas de iniciación al violín. Los resultados muestran que aquellos alumnos cuyos padres o tutores estuvieron más involucrados en el proceso de enseñanza-aprendizaje tuvieron mayores índices de motivación. Además, aquellos que se sintieron sobrepasados por las dificultades de las tareas propuestas derivadas de la secuenciación de contenidos estuvieron más desmotivados. Sin embargo, hubo excepciones debido a las individualidades de cada alumno.

Palabras clave: Educación musical, niño en edad preescolar, motivación, participación de los padres, instrumento musical.

\section{Abstract}

Content sequencing and the role of the family can provide a foundation for the beginning of instrumental learning, decisively influencing the students' motivation. This research focused on that influence with 11 preschool-aged violin students in private classes. For this purpose, an action-research was carried out by the teacher as an observing participant, using two didactic approaches in beginning violin. The results show that those students whose parents or caretakers were more involved in the teaching-learning process were more motivated. Also, students who felt overwhelmed by the difficulties of the proposed tasks derived from the content sequencing were less motivated. However, there were exceptions due to the individual personalities of each student.

Keywords: Music education, preschool children, motivation, parent participation, musical instrument.

\footnotetext{
${ }^{1}$ Este artículo se encuentra enmarcado en los resultados de la tesis doctoral de Roberto Macián: "Contraste de dos aproximaciones didácticas en la iniciación al violín: una investigación en el aula con niños de 4-5 años".
} 


\section{Introducción}

La motivación es "un proceso por el cual una actividad dirigida hacia un objetivo es instigada y mantenida" (Pintrich y Schunk, 1996, p. 4); además, es uno de los factores esenciales en el aprendizaje instrumental (Tripiana, 2016). Evaluar la motivación no es una tarea sencilla, aunque existe cierto consenso en que algunas conductas observables pueden denotar motivación: 1) elección de tareas, entre varias posibles; 2) esfuerzo, especialmente en tareas difíciles; 3) persistencia, dedicando más tiempo a determinadas actividades; y 4) logro, según los indicadores anteriores (Linnenbrink-Garcia et al., 2011; Pintrich y Schunk, 1996).

Según algunas investigaciones, la motivación es un proceso en el que se ven involucrados factores internos y externos del individuo. Entre los factores internos se encuentran: la personalidad, las creencias sobre uno mismo (autopercepción), los logros previos, los objetivos y la autoeficacia o el estado afectivo; mientras que se consideran factores externos: el entorno, las relaciones sociales, los modelos que tenga el alumno, la secuenciación de la enseñanza y las tareas que se le pidan (Austin et al., 2010; Hallam, 1998; Hallam, 2006; López de la Llave y Pérez-Llantada, 2006; Smith, 2011). Además, todos estos factores pueden influir en la motivación con el paso del tiempo (Sichivitsa, 2007; Tripiana, 2016).

Entre los factores externos, dos se consideran fundamentales para la motivación de los alumnos que asisten a clases de música o tocan un instrumento musical: la familia (Hurley, 1995; López de la Llave y Pérez-Llantada, 2006; Sichivitsa, 2007; Vasil, 2013) y la secuenciación de contenidos (Hallam, 1998; Smith, 2011).

La familia es especialmente relevante con los más jóvenes, ya que aportan estímulos musicales (Hallam, 2006) y ofrecen soporte práctico (como financiación o transporte) (Hallam, 1998). Sin embargo, también es importante que ofrezcan oportunidades para que perciban su compañía durante la práctica (acompañamiento parental) (Bugeja, 2009; Creech, 2010; Kalverboer, 2008). Según Bugeja (2009), existen dos formas de acompañamiento parental durante la práctica en clase; por un lado, la aproximación tradicional no contempla acompañamiento parental en clase, y por otro, la aproximación utilizada en el Suzuki permite y recomienda que los padres asistan a las sesiones (Starr, 2000).

El otro factor externo de vital importancia en la motivación de los alumnos (Smith, 2011) es la selección de las tareas en la secuenciación de contenidos (Hallam, 1998). Si la secuenciación no resulta adecuada y las tareas seleccionadas son demasiado sencillas o demasiado complejas, el alumno se puede desmotivar (Hallam, 1998).

Para Edmondson (2005) y Martín (2002) existen tres formas de organizar los contenidos técnicos en iniciación al violín: 1) Métodos de Mano Izquierda (MI), que inician al alumno en el manejo de la mano izquierda para producir los distintos sonidos del instrumento en pizzicato, retrasando durante un tiempo el inicio de la producción de sonido con el arco; 2) Métodos de Mano Derecha (MD), que se centran en el manejo del arco y la correcta emisión de sonido antes de abordar la producción de los distintos sonidos; y 3) Métodos de Ambas Manos (AM), que compaginan el desarrollo de la técnica de las dos manos de forma simultánea o combinada. Las tres aproximaciones presentan ventajas y desventajas en relación con el desarrollo técnico (Edmondson, 2005).

Los profesores deben tener en cuenta particularmente estos factores externos (acompañamiento familiar y secuenciación de contenidos). Si finalmente se produce, la desmotivación puede provocar el abandono de las clases; algunas causas de desmotivación son una práctica insuficiente, el sentimiento de incapacidad para la música, un menor logro respecto a los compañeros, la falta de apoyo familiar, ser menos hábil para seguir las instrucciones o la falta del sentimiento de recompensa en relación con el esfuerzo realizado (Hallam, 1998; López de la Llave y Pérez-Llantada, 2006).

Entre los estudios que han contrastado aproximaciones metodológicas distintas en la iniciación al violín se encuentran: Jensen (1990), quien aborda el estudio del agarre del arco; Lowe (1973) hace lo propio con la distribución de arco, mientras que Bergonzi (1997) y Miles (2010) estudian la entonación sobre el 
Influencia de la secuenciación de contenidos y el apoyo parental en la motivación de estudiantes de violín en edad preescolar

instrumento. Otros trabajos referidos al aprendizaje del violín por niños pequeños han estudiado el desarrollo de otras destrezas, pero no la motivación (Chantal, 2007; Zavalko, 2013). Por último, Koepp, (2004) y Kupresanin (2012) abordaron la secuenciación de contenidos técnicos y su adquisición por parte del alumnado. Dada la carencia de investigaciones previas, en este trabajo se estudian dos aproximaciones posibles en la secuenciación de contenidos y su influencia en la motivación de niños de 4 y 5 años, edad en la que se inician en el estudio del violín.

Los objetivos del trabajo fueron: 1) ayudar a mejorar la práctica docente del violín y 2) aportar conocimiento a la didáctica del violín en edades tempranas. Para ello, se buscó dar respuesta a las preguntas: 1) ¿cuáles son las actitudes ante dos aproximaciones didácticas con diferente secuenciación de contenidos?, y 2) ¿la asistencia de los familiares a las clases es relevante en los resultados de aprendizaje de los estudiantes?

La influencia de la secuenciación de contenidos y el acompañamiento parental en clase pueden influir en la motivación de los estudiantes muy jóvenes. La secuenciación de contenidos es un elemento importante de la intervención docente, por ello merece ser estudiada en profundidad con el fin de que el profesor tome decisiones informadas con relación a la temporización y gradación del contenido del aprendizaje para evitar la pérdida de motivación de niños pequeños.

\section{Método}

Para cumplir con los objetivos del estudio se llevó a cabo una investigación en aula, un estudio exploratorio de tipo etnográfico que aspira a convertirse en investigación-acción (IA) una vez realizado un segundo ciclo de intervención que proporcione datos para la triangulación. El método adoptado responde al objetivo de la investigación socio-crítica en tanto pretende cambiar la realidad -en este caso la de las personas que aprenden. El diseño aspira a obtener datos con el fin de ahondar en los problemas surgidos en los niños pequeños con la práctica del violín (Kemmis et al., 2014), intentando construir un cuerpo de conocimiento (Cohen y Manion, 2002) a partir de la sistematización de los datos obtenidos con el fin de mejorar cíclicamente la acción docente interpuesta. La investigación en aula la realizó el profesor (como investigador participante para mejorar su propia práctica) en un centro de titularidad pública de la provincia de Barcelona (Bisquerra, 2000; Cain, 2013; Kemmis y McTaggart, 1992).

La investigación se realizó en 30 sesiones individuales de 30 minutos a lo largo de un curso escolar, comprendiendo un único ciclo de IA. Asimismo, se realizaron 3 sesiones grupales de 45 minutos para ргерагаг un concierto al final del curso. Se recogieron datos de un total de 11 alumnos voluntarios del propio centro con edades comprendidas entre los 4 y los 5 años. Los participantes fueron agrupados por edades y asignados aleatoriamente a una de las dos aproximaciones didácticas (AM O MD) diseñadas por el investigador, las cuales constituyen las aproximaciones mayoritarias en los materiales didácticos publicados (Martín, 2002). De esto, se puede colegir que quizá éstas son las formas más eficaces de iniciación al violín. Además, es necesario mencionar aquí que no existen trabajos en la literatura científica que aborden un contraste de ambas aproximaciones, siendo éste el primero en considerarse como tal.

Con el fin de explicar la persistencia en la actividad violinística o la preferencia en la elección de tareas (ambos indicadores de motivación) se recogieron datos de matriculación de estos alumnos en las clases de violín durante los cuatro años posteriores a este ciclo IA. Estos datos consistieron en las altas y bajas en las clases de violín, causando posibles cambios de instrumento.

La recogida de datos se realizó mediante análisis de documentos, observación participante y entrevistas con padres y alumnos. Los documentos del centro (boletines de notas y programaciones didácticas) permitieron tener datos sobre las aptitudes y actitudes de los alumnos que la participaron de forma voluntaria. Para recoger datos de observación se utilizó un diario de campo a lo largo de todas las sesiones realizadas. Además de las 30 sesiones de adiestramiento mencionadas, se realizaron tres sesiones de grabaciones en video de las interpretaciones de los alumnos coincidiendo con la última semana de cada trimestre. Los registros en video presentan las ventajas de permanencia y densidad (Grimshaw, 1982), y se completaron con otro tipo de registros, como la observación (Bottorff, 2005). 
Influencia de la secuenciación de contenidos y el apoyo parental en la motivación de estudiantes de violín en edad preescolar

Las entrevistas con los familiares fueron semiestructuradas (Bisquerra, 2000; Elliott, 1989) y se realizaron durante los días de grabación, quedando también registradas en video. A los alumnos se les hizo una única entrevista en la última sesión del curso. En este caso fue más estructurada para obtener respuesta, pues la falta de verbalización de los alumnos tan jóvenes podría producir problemas en la precisión de la información recogida (Cameron, 2005). Así, a los familiares se les formularon, respectivamente, 13, 9 y 11 preguntas en las tres sesiones, indagando en los comportamientos y motivaciones de los alumnos. A los alumnos se les formularon cuatro preguntas breves sobre sus gustos en cuanto a las actividades y su deseo de continuar tocando el violín en el siguiente curso.

Dado el diseño de investigación adoptado, los datos recogidos fueron de tipo cualitativo en su mayoría. No obstante, también se tomaron algunos datos de tipo cuantitativo. Los datos provenientes de todos los registros se analizaron con el programa de análisis de datos cualitativos Atlas-ti.

\subsection{Categorías de análisis}

Como se ha comentado, la motivación puede inferirse a partir de las conductas observables de los niños concretadas en un conjunto de indicadores según los comportamientos del alumnado. Por tanto, se definió una categoría previa denominada Motivación, en la que emergieron los distintos indicadores de ésta a partir de los datos analizados. Además, los indicadores permitieron establecer los niveles aproximados de motivación de cada alumno a lo largo del curso (véanse los indicadores y sus agrupaciones en categorías en la sección de Resultados). Otra categoría previa fue el Apoyo parental en clase, recogiéndose datos de asistencia de familiares según: 1) Asistencia, cuando los familiares asistieron a la sesión de clase completa; 2) Asistencia parcial, si asistieron durante un determinado período de tiempo de la clase; y 3) Ausencia, cuando los familiares no asistieron.

\subsection{Diseño de la intervención docente}

Se diseñaron dos acciones didácticas diferenciadas en función de cada aproximación didáctica (AM o MD), según las características del centro, aula y tiempos docentes (Campá, 2012; Chantal, 2007). Además, se adoptaron y utilizaron los objetivos y contenidos de algunos métodos vigentes (Hamann y Gillespie, 2004; Rolland, 1985; Starr, 2000), teniendo en cuenta la legislación catalana vigente en el Decret 179/1993, de 27 de julio (Generalitat de Catalunya, 1993). ${ }^{2}$

La metodología docente adoptó el modelo en tres pasos de Price (1989): 1) ofrecer información al alumno; 2) recibir la respuesta del alumno sobre esta información; y 3) ofrecer un feedback al alumno sobre esta respuesta. Así, se realizaron cuatro tipos de actividades: 1) práctica y experimentación con el instrumento con base en los objetivos y contenidos propuestos; 2) montaje, desmontaje y mantenimiento del instrumento, tal como se recomienda en la bibliografía (Galka, 2011; Hamann y Gillespie, 2004); 3) concienciación corporal con o sin el instrumento, basada en la propuesta de Kovács y Pásztor (2010); y 4) trabajo de las canciones sin instrumento, según la práctica habitual en la Escuela de Música donde se ha realizado la investigación.

Los materiales de la acción docente fueron diseñados en función de las obras didácticas consultadas y de acuerdo a la edad de los niños que participaron en el estudio (Anderson y Frost, 1985; Blackwell y Blackwell, 1998; McPherson y Davidson, 2010; Rolland, 2000). Se dividieron los contenidos a trabajar con los alumnos para facilitar el diseño de los materiales. Por un lado, se debía tocar en las cuatro cuerdas del violín (sol, re, la y mi) y, por otro, tocar con los tres dedos de la mano izquierda (0-1-23). Así, los alumnos podrían ejecutar 16 sonidos, cuatro sonidos en cada cuerda. Por cuestiones de desarrollo de los alumnos y de disponibilidad temporal, no se llegó a practicar con el cuarto dedo.

Para la aproximación MD se tomó como base el manejo del arco con distintos patrones rítmicos en las diferentes cuerdas. A partir de ahí se incluyó la ejercitación de cada dedo de mano izquierda por

\footnotetext{
${ }^{2}$ Las regiones españolas (Comunidades Autónomas) tienen las competencias regulatorias en materia de educación, tanto general como específica.
} 
separado, dedicando cierto tiempo para practicarlo en todas las cuerdas antes de pasar a utilizar otro dedo. Por otro lado, la aproximación Am se fundamentó en el manejo simultáneo de ambas manos, practicando un único ejercicio preparatorio para el manejo del arco. Posteriormente, se practicó con cada dedo sobre una única cuerda, comenzando en la cuerda re. En ambas aproximaciones, una vez trabajando nuevos sonidos, se podían combinar con sonidos ya estudiados.

En el diseño de las acciones docentes se emplearon canciones populares adaptadas a cada secuencia didáctica en la medida de lo posible. Las canciones populares se adaptaron a cada una de las secuencias didácticas, ya que están ampliamente recomendadas en la bibliografía escolástica para ser utilizadas en el aprendizaje instrumental por alumnos preescolares (Loong, 2007; Miyamoto, 2007; Zubeldia y Díaz, 2010). Cuando no se pudo, el profesor propuso piezas adecuadas a las secuencias didácticas, dicho de otro modo, adaptadas a los sonidos que conocían los alumnos y los que se debían practicar para seguir una secuencia lógica de aprendizaje del violín. La propuesta constituyó una colección de 25 ejercicios/canciones para cada aproximación. Ambas comenzaron con la misma pieza y, tras seguir su correspondiente secuencia didáctica, se llegó a otra pieza que resumió todos los contenidos trabajados, idéntica en ambas aproximaciones.

Por último, dado que este curso no era de enseñanza reglada, no se realizó una evaluación cuantitativa de tipo selectivo. Sólo se observó el progreso de los alumnos siguiendo los diferentes criterios técnicos provenientes de la literatura didáctica del instrumento sobre la colocación y manejo del cuerpo e instrumento. Este conjunto de criterios fue aplicado en ambas aproximaciones.

Para la colocación del instrumento y el cuerpo se tuvo en cuenta: 1) Posición del violín entre la mandíbula y la clavícula sin elevación del hombro (Hoppenot, 2005); 2) Apertura del brazo 45 grados desde el centro del eje del cuerpo (Havas, 2003; Starr, 2000) y 90 grados respecto a la vertical (Lee, 2003); 3) Posición del botón del violín apuntando al centro del cuello (Rolland, 2000) y sin ejercer demasiada presión (Lee, 2003); y 4) Colocación de la cabeza a medio camino entre recta y sobre el instrumento (Flesch, 2000), con las piernas ligeramente separadas, las puntas de los pies abiertas y sin bloquear las rodillas (Flesch, 2000; Rolland, 1985).

Para la mano izquierda se observó: 1) Posicionamiento recto de la muñeca en relación con la mano y antebrazo (Galamian, 1998; Lee, 2003); 2) Colocación de los dedos según su longitud para permitir la pisada con la yema del dedo y formando un arco (Lee, 2003); 3) Posición del codo debajo del violín (Havas, 1998), más cerca del cuerpo en cuerda mi y más alejado en cuerda sol (Lee, 2003); 4) Colocación del pulgar en oposición al índice (Galamian, 1998), sin que sobresalga por encima de las cuerdas (Lee, 2003); 5) pisar con la yema del dedo, evitando la zona demasiado cercana a la uña (Lee, 2003); y 6) dejar los dedos inactivos relajados y cercanos a la cuerda, manteniendo los dedos una vez pisan la cuerda (Lee, 2003).

Los criterios utilizados para la mano derecha fueron: 1) "Agarre ecléctico" (Jensen, 1990) ${ }^{3}$; 2) Movimiento de paso de arco accionando el codo (Cohen, 1996), acotando el movimiento a la mitad superior (Lee, 2003) mediante el empleo de marcas visibles (Starr, 2000); 3) Cambio de cuerdas subiendo o bajando el codo (Lee, 2003); 4) Punto de contacto intermedio entre el puente y el diapasón (Flesch, 1995); y 5) Indicaciones sencillas sobre los factores que condicionan la producción de sonido (velocidad-presiónpunto de contacto), utilizando correcciones a los errores puntuales, pero sin entrar en detalle en cómo afecta cada uno de estos factores al resto.

También se tomaron dos decisiones de diseño: 1) Impedir la práctica de los alumnos con el instrumento en sus casas durante el primer trimestre (The University of the State of New York, 1971) y 2) Dejar que la asistencia a clase de los familiares fuera voluntaria (Bugeja, 2009).

3 Este agarre corresponde al más eficaz y con menor tensión: pulgar arqueado debajo del dedo corazón formando un círculo, el contacto del pulgar en la vara en el punto más cercano a la nuez, el índice se posa sobre la vara a la altura de la falange intermedia, dedos anular y corazón pegados, índice ligeramente separado de los otros dedos y el meñique arqueado sobre la vara en la cara interna de ésta. 


\section{Resultados}

Realizado el análisis de los datos procedentes de la observación, surgieron un total de 18 indicadores de motivación (ver figura 1). De los indicadores observados, 5 constituyeron el $50 \%$ del total de las incidencias observadas en los alumnos (indicadores 1 a 5 ). Otros 5 fueron también frecuentes (indicadores 6 a 10). El resto de indicadores (11 a 18) fueron minoritarios.

De los indicadores mayoritarios (1 a 5), la aproximación AM tuvo mayor incidencia en el indicador Negarse a tocar en clase. De los siguientes indicadores más numerosos (6 a 10), la aproximación Am tuvo mayor incidencia en: Deseo de abandonar las clases de violín y Reticencia a asistir a clase. De hecho, la mitad de los alumnos AM mostraron estos indicadores en alguna ocasión. Algunos alumnos Am tuvieron clara su decisión de continuidad o abandono. Otros, después de dudar sobre esta cuestión, adoptaron una respuesta en cuanto a deseo de continuidad o abandono, pero no sucedió con todos los alumnos.

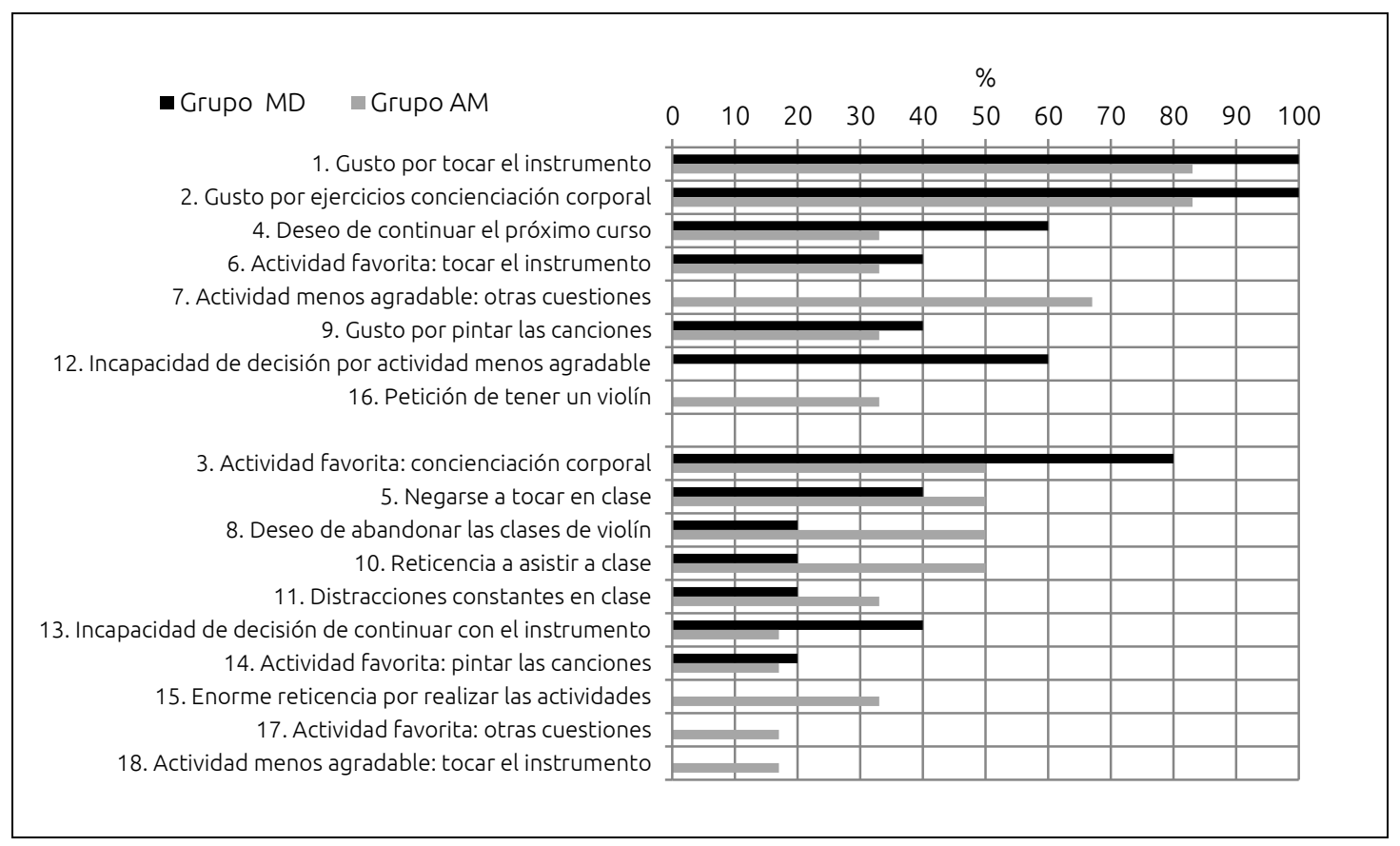

Figura 1. Indicadores de motivación numerados según incidencia (divididos en positivos-negativos)

Se recogieron datos relevantes de motivación relacionados con dos alumnos Am que presentaron grandes resistencias a trabajar. Así, algunas actividades fueron desagradables para estos alumnos, cosa que no ocurrió con el grupo MD. Por otro lado, también resultó relevante que los únicos niños que pidieron disponer de su propio violín antes de tiempo fueron algunos del grupo AM.

De los 5 indicadores mayoritarios (1 a 5), la aproximación MD tuvo más incidencia en indicadores positivos de motivación: Gusto por tocar el instrumento y Deseo de continuar el próximo curso. De los siguientes más comunes (6 a 10), la aproximación MD tuvo mayor incidencia en Actividad favorita: tocar el violín. También resulta destacable que los alumnos MD no señalaron ninguna actividad desagradable. Otro dato relevante es que más de la mitad de los alumnos MD manifestó el deseo de continuar tocando el violín.

Según aparecieron los indicadores a lo largo del curso y tal y como se trianguló con los datos obtenidos en las distintas entrevistas, se pudo establecer la tendencia en el nivel de motivación que tuvieron los alumnos: alto, creciente, muy cambiante, decreciente o bajo (ver figura 2). Para ello, se distinguió entre indicadores positivos e indicadores negativos. Cuando se observaron más indicadores positivos que negativos en una misma sesión de clase, se consideró que el alumno tuvo un nivel de motivación "Alto". 
Influencia de la secuenciación de contenidos y el apoyo parental en la motivación de estudiantes de violín en edad preescolar

Cuando se observaron más negativos que positivos, se consideró que el alumno tuvo un nivel de motivación "Bajo". Sin embargo, en muchas sesiones no se pudo establecer una diferencia notable entre los indicadores negativos y positivos; en esos casos, se consideró al alumno motivado o desmotivado según la última referencia clara sobre la motivación.

Las fluctuaciones entre los niveles "Alto" y "Bajo" permitieron trazar las líneas de tendencia aproximadas a lo largo del curso. A continuación se presentan algunos ejemplos claros en cuanto a los niveles de motivación de los alumnos.

Algunos padres percibieron niveles altos de motivación en sus hijos:

Cuando no se porta bien, lo "amenazamos" (haciendo el gesto de entrecomillado con los dedos) con que no vendrá a la clase de violín... Y así cambia enseguida de comportamiento [...]. Le encanta la clase... (Registro en video, entrevista a familiar de alumno $7 \mathrm{AM}$ ).

De igual manera, el alumno 6AM también mostró una elevada motivación desde la primera sesión. En ocasiones, al hacerle alguna corrección no dejaba de tocar el instrumento, tratando de resolver los problemas por sí mismo. Una situación similar presentó el alumno 8MD.

El alumno 10Am representa un ejemplo de motivación creciente. En el primer trimestre, se anotó:

El alumno muestra pocas ganas, alegando que no tiene energía. [...] El padre comenta que el horario de clase coincide con su horario habitual de comida [...] Se ha negado a tocar y ha querido terminar la clase antes de tiempo. (Diario de campo, anotaciones sobre alumno 10AM).

Además de un mayor esfuerzo en algunos alumnos, se deduce de sus comentarios que proyectaban un punto óptimo de motivación para abordar el siguiente curso:

- ¿Y qué me dices de tocar el violín?, ¿te gusta mucho?

— Mucho no, iimuchísimo!!... (Registro en video, entrevista a alumno 10AM).

Los alumnos 9MD y 2MD también experimentaron este incremento. El alumno 9MD al cambiar su horario y realizar las sesiones más temprano. El alumno 2MD a lo largo del primer trimestre, llegando a ser una de las alumnas más implicadas hacia final de curso. El alumno $11 \mathrm{MD}$ fue el alumno que más altibajos presentó en cuanto a motivación, alternando momentos de gran interés con otros de desgana, llegando a negarse a tocar en alguna ocasión en el segundo trimestre.

Por otro lado, algunos alumnos experimentaron una pérdida de motivación. El ejemplo más claro fue el alumno 3AM. En las primeras sesiones, el alumno parecía muy entusiasmado con las novedades que le ofrecían las clases, lo que confirmaban los familiares.

Viene muy a gusto [...] A veces se duerme en el coche cuando viene del colegio [...] y tal vez por eso puede venir un poco cansado... Pero... En principio, viene con muchas ganas... (Registro en video, entrevista a familiares del alumno 3AM).

Después de un tiempo prolongado de pérdida de interés, el alumno confirmó su total desmotivación:

— ¿Y qué es lo que menos te gusta de las clases?

- Tocar el violín.

- ¿De verdad?

— ¡Que sí!... (Registro en video, entrevista a alumno 3AM)

Pero este no fue el único caso. El alumno 1 Am también mostró un descenso en la motivación a lo largo del curso, pasando de mostrar interés a negarse a practicar en las últimas clases. A diferencia del alumno 3AM, fue un cambio más gradual. Finalmente, los alumnos 4AM y 5AM ofrecieron numerosas muestras de 
desmotivación durante todo el curso, mostrando ligeras mejoras a en momentos muy puntuales. Según los familiares, el alumno 4AM era muy autoexigente y no toleraba errores en las actividades, mientras que el alumno 5Am ofreció dudas al profesor sobre que pudiese terminar el curso. Estos datos sugieren que la motivación se vio favorecida en la aproximación MD.

\subsection{Apoyo parental en clase}

La asistencia de los familiares fue diferente en cada caso (ver figura 2), contabilizándose en qué sesiones concretas asistieron a las clases y el tiempo que permanecieron en el aula según se ha especificado en el apartado de categorías de análisis. 


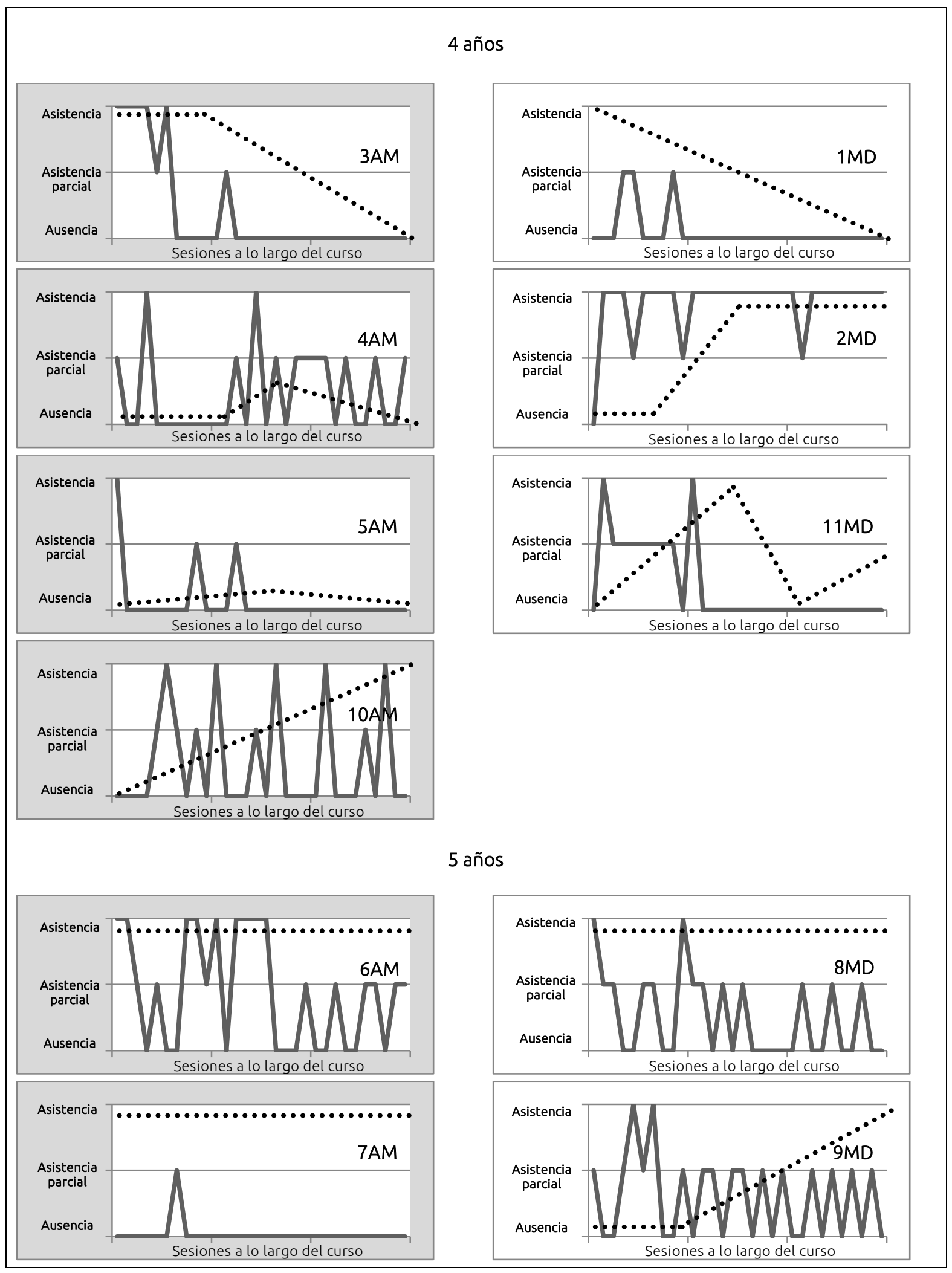

Figura 2. Asistencia de familiares (línea continua) y nivel de motivación (línea de puntos) 
Influencia de la secuenciación de contenidos y el apoyo parental en la motivación de estudiantes de violín en edad preescolar

Hubo tres alumnos que manifestaron su discrepancia en la asistencia de los familiares a las clases: alumno $11 \mathrm{MD}$, alumno 3AM, alumno 4AM y alumno 5AM. El olvido de los materiales por los alumnos fue un indicador importante de la toma de responsabilidad de los padres en el aprendizaje de los hijos. Este factor de responsabilidades es crucial en la motivación de los estudiantes. Pese a la importancia atribuida por el profesor, algunos alumnos no llevaron sus materiales en varias ocasiones: alumno 5 AM (9 ocasiones), alumno 1MD (6), alumno 4AM (3) y alumno 3AM (2). Dada la corta edad de los alumnos, esta cuestión debía tenerse en cuenta por parte de padres o tutores.

\subsection{Tasas de abandono del instrumento}

El profesor tuvo dudas en cuanto a la finalización del curso por parte de dos alumnos Am. Sin embargo, todos los alumnos continuaron con las clases durante otro curso posterior al del trabajo de campo en el aula. Tras dos cursos académicos, la mitad de los alumnos Am dejó definitivamente las clases de violín. Sin embargo, sólo el alumno 9MD dejó las clases después de dos cursos, retomando las clases de violín posteriormente. Dos alumnos dejaron las clases después del tercer curso académico en MD, coincidiendo con el año en el que tenían acceso a probar otros instrumentos.

\section{Discusión}

Los resultados de este trabajo confirman los de otras investigaciones (Hurley, 1995; Vasil, 2013) y coinciden con ciertos presupuestos metodológicos de algunos manuales de aprendizaje instrumental (Hallam, 1998; Hallam, 2006; López de la Llave y Pérez-Llantada, 2006), mostrando que la motivación es una mezcla de factores internos y externos del individuo. Además, los resultados sugieren que la motivación es un proceso dinámico, cuestión ya apuntada por Sichivitsa (2007) y Tripiana (2016).

Si bien es cierto que los factores externos han podido resultar fundamentales, al menos en un caso los resultados apuntan a una mayor fuerza de la motivación interna o intrínseca: este alumno mostró elevada motivación todo el curso pese a no recibir gran apoyo de su familia. También se observó esfuerzo y persistencia a la hora de tocar algunas canciones en algunos alumnos, indicadores de motivación apuntados en la introducción de este trabajo y que consolidan los resultados de otros trabajos (Linnenbrink-Garcia et al., 2011; Pintrich y Schunk, 1996).

\subsection{Sobre el apoyo parental}

Como se mencionó antes, y a diferencia de lo que se recomienda en la bibliografía, tres alumnos AM y uno MD se negaron a que sus familiares asistieran a las clases (Bugeja, 2009; Creech, 2010; Kalverboer, 2008). Esto podría deberse a una sensación de falta de control en las actividades tocando el instrumento, tratando de evitar así una desaprobación por parte de los familiares. Este motivo aparece en las referencias consultadas como causante de la pérdida de motivación y, en casos extremos, de abandono de las clases (Hallam, 1998; Hurley, 1995; López de la Llave y Pérez-Llantada, 2006).

El olvido del libro de materiales es un indicador de la responsabilidad paterna hacia el aprendizaje de los niños, que está en relación con la motivación social, un factor externo en el niño. Dada la corta edad de los alumnos, esta cuestión dependía de los familiares (Hallam, 1998, 2006). Los alumnos podrían haber entendido este hecho como una falta de interés de los familiares o que no le diesen importancia a la clase de violín, influyendo negativamente en la motivación (Hallam, 1998; Hurley, 1995; Vasil, 2013).

En términos generales, independientemente de los resultados técnicos del alumnado, los resultados muestran que hubo más problemas de motivación cuando los alumnos no dispusieron de acompañamiento familiar en las clases de violín (Bugeja, 2009; Creech, 2010; Kalverboer, 2008), especialmente relevante en AM. Por tanto, cuando no se dispone de acompañamiento, los resultados sugieren que los niños de estas edades deberían iniciarse en el estudio del violín mediante la aproximación MD. 


\subsection{Sobre la secuenciación de contenidos}

Al menos en un caso (alumna AM), el desánimo por los resultados obtenidos debido a una elevada autoexigencia pudo ser la causa de la falta de motivación mostrada en las clases (López de la Llave y PérezLlantada, 2006). La realización de tareas complejas de forma simultánea pudo ser la causa de esta pérdida de interés (Edmondson, 2005). De ser así, los alumnos de estas edades deberían abordar una sola tarea en cada momento, soslayando secuencias didácticas con una demanda cognitiva alta que no puedan superar con el fin de evitar la desmotivación (Edmondson, 2005; Hallam, 1998; Smith, 2011). Quizá las secuencias $\mathrm{MD}$, en las que se pueden realizar las tareas de forma individualizada, serían más adecuadas con los alumnos de corta edad de acuerdo a los resultados de motivación obtenidos. Esto fue especialmente relevante en los alumnos de 4 años, observando menores niveles de motivación en los alumnos Am.

\subsection{Sobre el abandono de las clases}

Es destacable que el grupo Am tuvo una mayor tasa de abandono después de dos cursos y la aproximación MD después de tres. Estos datos apuntan a la elección de tareas (secuenciación de contenidos) como influyentes en la motivación (Edmondson, 2005; Smith, 2011) y, tal y como se apunta anteriormente, resultan adecuados como indicadores de motivación (Linnenbrink-Garcia et al., 2011; Pintrich y Schunk, 1996).

Este menor nivel de motivación de los alumnos Am quizá pudo deberse a que se sintieran menos capaces en la realización de las tareas musicales propuestas o no pudieron seguir las instrucciones correctamente (Hallam, 1998; López de la Llave y Pérez-Llantada, 2006; Smith, 2011). Esto está directamente relacionado con la secuenciación de contenidos técnicos, encontrando que se debería prestar más atención a este motivador externo (Edmondson, 2005; Hallam, 1998; Smith, 2011).

En el caso de los alumnos MD, el curso de abandono mayoritario coincidió con el curso en el que pudieron probar otros instrumentos. Es posible que el deseo de cambiar a un instrumento con una menor demanda de recursos cognitivos y de dedicación de tiempo pudiera haber forzado la decisión de abandonar el violín por parte de los alumnos más desmotivados (Hallam, 1998; Linnenbrink-Garcia et al., 2011; Pintrich y Schunk, 1996). Sin embargo, este es un cuestionamiento que no fue abordado en este estudio y que merecería estudiarse en un trabajo posterior.

A modo de conclusión, y de acuerdo con los datos obtenidos, la motivación externa y la demanda cognitiva (derivada de las tareas requeridas en las aproximaciones) supusieron factores determinantes en la motivación, especialmente con los alumnos más jóvenes. No obstante, en algunos casos vistos en este trabajo la motivación intrínseca podría haber suplido las carencias de motivación externa. Con todo, dada la naturaleza de la presente investigación, estos datos deben ser tomados con cautela, debiendo llevarse a cabo ciclos consecutivos para obtener más datos con los que se puedan triangular los obtenidos para ofrecer resultados más concluyentes.

\section{Referencias}

Anderson, G. E. y Frost, R. S. (1985). All for strings: comprehensive string method (Vol. 1). Neil A. Kjos Music Company.

Austin, J., Renwick, J. y McPherson, G. E. (2010). Developing motivation. En G. E. McPherson (Ed.), The child as musician: a handbook of musical development (pp. 213-238). Oxford University Press.

Bergonzi, L. (1997). Effects of finger markers and harmonic context on performance of beginning string students. Journal of Research in Music Education, 45(2), 197-211. https://doi.org/10.2307/3345580

Bisquerra, R. (2000). Métodos de investigación educativa. Guía práctica. Ceac. 
Influencia de la secuenciación de contenidos y el apoyo parental en la motivación de estudiantes de violín en edad preescolar

Blackwell, K. y Blackwell, D. (1998). Fiddle time starters: a beginner book for the young violinist. Oxford University Press.

Bottorff, J. L. (2005). El uso de las grabaciones de vídeo en la investigación cualitativa. En J. M. Morse (Ed.), Asuntos críticos en los métodos de educación cualitativa (pp. 309-329). Universidad de Alicante.

Bugeja, C. (2009). Parental involvement in the musical education of violin students: Suzuki and "traditional" approaches compared. Australian Journal of Music Education, 1, 19-28.

https://eric.ed.gov/?id=EJ912407

Cain, T. (2013). Investigación-acción en educación musical. En M. Díaz, y A. Giráldez (Eds.), Investigación cualitativa en educación musical (pp. 57-75). Graó.

Cameron, H. (2005). Asking the tough questions: a guide to ethical practices in interviewing young children. Early Child Development and Care, 175(6), 597-610.

https://doi.org/10.1080/03004430500131387

Campá, C. M. (2012). Propuesta metodológica para la enseñanza de la clase de conjunto en el grado elemental aplicada al violoncello (Tesis doctoral no publicada). Universidad Rey Juan Carlos, Madrid.

Chantal, K. (2007). O ensino de violino no estágio pré-operacional: um estudo piloto sobre o desenvolvimento técnico, com considerações da psicologia cognitiva e do desenvolvimento [La enseñanza del violín en la etapa preoperativa: un estudio piloto sobre desarrollo técnico, con consideraciones de psicología cognitiva y del desarrollo](Tesis de máster no publicada). Universidade Federal de Minas Gerais, Belo Horizonte.

Cohen, E. (1996). Eta Cohen's violin method. Student's book (Vol. 1). Novello Publishing Limited.

Cohen, L. y Manion, L. (2002). Investigación en la acción. En M. A. Casanova (Dir.), Métodos de investigación educativa (pp. 272-298). La Muralla.

Creech, A. (2010). Learning a musical instrument: the case for parental support. Music Education Research, 12(1), 13-32. https://doi.org/10.1080/14613800903569237

Edmondson, R. (2005). Beginning violin: pizzicato vs. arco. American String Teacher, 55(1), 50-53.

https://doi.org/10.1177/000313130505500107

Elliott, J. (1989). "Action-research": normas para la autoevaluación en los colegios. En L. Haynes (Comp.), Investigación/acción en el aula (pp. 21-48). Generalitat Valenciana.

Flesch, C. (1995). Los problemas del sonido en el violín. Real Musical.

Flesch, C. (2000). The art of violin playing (Vol. 1). Carl Fischer.

Galamian, I. (1998). Interpretación y enseñanza del violín. Ediciones Pirámide.

Galka, C. (2011). Violín básico. Nueva Carish España.

Generalitat de Catalunya. (1993). Decret 179/1993, de 27 de juliol, pel qual es regulen les escoles de música i dansa [Decreto 179/1993, de 27 de julio, por el que se regulan las escuelas de música y danza]. (DOGC 1779, 4 de agosto de 1993). https://goo.gl/YVzT2b

Grimshaw, A. D. (1982). Sound-image data records for research on social interaction. Sociological Methods \& Research, 11(2), 121-144. https://doi.org/10.1177/0049124182011002002 
Influencia de la secuenciación de contenidos y el apoyo parental en la motivación de estudiantes de violín en edad preescolar

Hallam, S. (1998). Instrumental teaching: A practical guide to better teaching and learning (1a. ed.). Heinemann.

Hallam, S. (2006). Music psychology in education. University of London.

Hamann, D. L. y Gillespie, R. (2004). Strategies for teaching strings: building a successful string and orchestra program. Oxford University Press.

Havas, K. (1998). A new approach to violin playing. Bosworth.

Havas, K. (2003). The twelve lesson course in a new approach to violin playing. Bosworth.

Hoppenot, D. (2005). El violín interior. Real Musical.

Hurley, C. G. (1995). Student motivations for beginning and continuing/discontinuing string music instruction. The Quarterly Journal of Music Teaching and Learning, 6(1), 44-55.

Jensen, J. L. (1990). A comparison of initial violin bow hold approaches in undergraduate string techniques classes, including assessments of the influence of baseline ratings of subjects' finger dexterity and mental images of the violin bow hold. Dialogue in Instrumental Music Education, 14(1), 10-34.

Kalverboer, K. (2008). Parental involvement in private violin lessons: survey of teacher attitudes and practices (Tesis de máster no publicada). McGill University, Montreal.

Kemmis, S., McTaggart, R. y Nixon, R. (2014). The action research planner: doing critical participatory action research. https://doi.org/10.1007/978-981-4560-67-2

Kemmis, S. y McTaggart, R. (1992). Cómo planificar la investigación-acción. Laertes.

Koepp, R. A. (2004). The effect of using a two-string teaching method versus a four-string teaching method on the performance of beginning fourth-grade orchestra students (Tesis de máster). ProQuest Dissertations \& Theses (UMI No. 1420529)

Kovács, G. y Pásztor, Z. (2010). Ejercicios preparatorios para instrumentistas (método Kovács) (1a. ed.). Graó.

Kupresanin, D. (2012). The effect of string instruction method books on left hand shape of beginning violinists and violists (Tesis de master no publicada). Texas Tech University: Lubbock. https://ttu-ir.tdl.org/ttuir/handle/2346/46977

Lee, H. A. (2003). Towards a dynamic pedagogy: contemporary pedagogical approaches to basic violin technique (Tesis doctoral). ProQuest Dissertations \& Theses. (UMI No. 3091026)

Linnenbrink-Garcia, L., Maehr, M. L. y Pintrich, P. R. (2011). Motivation and achievement. En R. Colwell y P. R. Webster (Eds.), MENC handbook of research on music learning (Vol. 1: Strategies) (pp. 216-264). Oxford University Press.

Loong, C. (2007). Early childhood music: Materials and activities. Criteria and guidelines for selecting and using materials in early childhood music lessons. Kodaly Envoy, 33(4), 5-8.

https://www.cyloong.com/bio/professional/

López de la Llave, A. y Pérez-Llantada, M. C. (2006). Psicología para intérpretes artísticos. Thomson.

Lowe, H. L. (1973). A study of the tone quality of beginning violin students using the long bow-stroke approach as compared to the short bow-stroke approach (Tesis doctoral no publicada). Ball State University. http://cardinalscholar.bsu.edu/handle/handle/177830 
Influencia de la secuenciación de contenidos y el apoyo parental en la motivación de estudiantes de violín en edad preescolar

Martín, W. (2002). Historia, literatura, pedagogía y cultura de la viola. Temario completo para oposiciones. Musicalis.

McPherson, G. E. y Davidson, J. W. (2010). Playing an instrument. En G. E. McPherson (Ed.), The child as musician: a handbook of musical development (pp. 331-351). Oxford University Press.

Miles, B. (2010). Effects of finger placement markers on intonation for beginning string players (Tesis de master no publicada). University of Florida. https://ufdc.ufl.edu/AA00000349/00001

Miyamoto, K. A. (2007). Musical characteristics of preschool-age students: A review of literature. UpdateApplications of Research in Music Education, 26(1), 26-40.

https://doi.org/10.1177/87551233070260010104

Pintrich, P. R. y Schunk, D. H. (1996). Motivation in education: theory, research, and applications. Merrill.

Price, H. E. (1989). An effective way to teach and rehearse: research supports using sequential patterns. Update: Applications of Research in Music Education, 8(1), 42-46.

https://doi.org/10.1177/875512338900800110

Rolland, P. (1985). Young strings in action: Paul Rolland's approach to string playing. Teacher's book (Vol. 1). Boosey \&Hawkes.

Rolland, P. (2000). Basic principles of violin playing. Alfred Music Publishing.

Sichivitsa, V. O. (2007). The influences of parents, teachers, peers and other factors on students' motivation in music. Research Studies in Music Education, 29(1), 55-68.

https://doi.org/10.1177/1321103X07087568

Smith, B. P. (2011). Motivation to learn music: a discussion of some key elements. En R. Colwell, y P. R. Webster (Eds.), MENC handbook of research on music learning (Vol. 1: Strategies) (pp. 265-299). Oxford University Press.

Starr, W. (2000). The Suzuki violinist: a guide for teachers and parents. Alfred Music Publishing.

The University of the State of New York (1971). Teaching strings. Albany: The University of the State of New York. https://eric.ed.gov/?id=ED056052

Tripiana, S. (2016). Estrategias de motivación durante el aprendizaje instrumental. Revista Internacional de Educación Musical, 4, 25-33. https://doi.org/10.12967/RIEM-2016-4-p025-033

Vasil, M. (2013). Extrinsic motivators affecting fourth-grade students' interest and enrollment in an instrumental music program. Update: Applications of Research in Music Education, 32(1), 74-82.

https://doi.org/10.1177/8755123313502345

Zavalko, K. (2013). Teaching preschoolers playing the violin on the basis of innovative violin techniques. Problems in Music Pedagogy, 12, 59-67. http://pmp.du.lv/wp-content/uploads/2017/07/PMP 2013 Vol12.pdf

Zubeldia, M. y Díaz, M. (2010). Los métodos para el estudio del violonchelo en la etapa inicial: análisis comparativo. CiDd: II Congrés Internacional de Didàctiques 2010 (pp. 1-6). 